\title{
MuSK autoantibodies in myasthenia gravis detected by cell based assay - A multinational study
}

\author{
A.I. Tsonis ${ }^{\text {a,b }}$, P. Zisimopoulou ${ }^{\text {a,* }}$, K. Lazaridis a , J. Tzartos ${ }^{\text {a }}$, E. Matsigkou ${ }^{\text {a }}$, V. Zouvelou ${ }^{\text {c }}$, R. Mantegazza ${ }^{\text {d }}$, \\ C. Antozzi ${ }^{d}$, F. Andreetta ${ }^{d}$, A. Evoli e, F. Deymeer ${ }^{f}$, G. Saruhan-Direskeneli ${ }^{\text {f }}$, H. Durmus ${ }^{\mathrm{f}}$, T. Brenner ${ }^{\mathrm{g}}$, \\ A. Vaknin ${ }^{g}$, S. Berrih-Aknin ${ }^{\mathrm{h}}$, A. Behin ${ }^{\mathrm{h}}$, T. Sharshar $^{\mathrm{i}}$, M. De Baets ${ }^{\mathrm{j}}$, M. Losen ${ }^{\mathrm{j}}$, P. Martinez-Martinez $^{\mathrm{j}}$, \\ K.A. Kleopa ${ }^{k}$, E. Zamba-Papanicolaou ${ }^{k}$, T. Kyriakides ${ }^{k}$, A. Kostera-Pruszczyk ${ }^{1}$, P. Szczudlik ${ }^{1}$, B. Szyluk ${ }^{1}$, \\ D. Lavrnic ${ }^{\mathrm{m}}$, I. Basta ${ }^{\mathrm{m}}$, S. Peric ${ }^{\mathrm{m}}$, C. Tallaksen ${ }^{\mathrm{n}, \mathrm{o}}$, A. Maniaol $^{\mathrm{n}}$, C. Casasnovas Pons $^{\mathrm{p}}$, J. Pitha ${ }^{\mathrm{q}}$, M. Jakubíkova ${ }^{\mathrm{q}}$, \\ F. Hanisch ${ }^{\mathrm{r}}$, S.J. Tzartos ${ }^{\mathrm{a}, \mathrm{b}, \mathrm{s}, *}$
}

a Hellenic Pasteur Institute, Athens, Greece

${ }^{\mathrm{b}}$ University of Patras, Patras, Greece

c Neurology Department, Aeginition Hospital, Athens, Greece

d Neurological Institute "C. Besta", Milano, Italy

e Institute of Neurology, Catholic University, Rome, Italy

${ }^{\mathrm{f}}$ Istanbul University, Istanbul, Turkey

${ }^{g}$ Hadassah Hebrew University Medical Center, Jerusalem, Israel

${ }^{\mathrm{h}}$ UPMC and INSERM, Paris, France

i Raymond Poincaré Hospital, Garches, France

j School for Mental Health and Neuroscience, Maastricht University, The Netherlands

${ }^{\mathrm{k}}$ The Cyprus Institute of Neurology and Genetics, Nicosia, Cyprus

${ }^{1}$ Department of Neurology, Medical University of Warsaw, Poland

${ }^{\mathrm{m}}$ Neurology Clinic, Clinical Center of Serbia, School of Medicine, University of Belgrade, Belgrade, Serbia

${ }^{\mathrm{n}}$ Norway Department of Neurology, Ullevaal University Hospital, Oslo, Norway

${ }^{\circ}$ Faculty of Medicine, Olso University, Norway

P Bellvitge University Hospital, Barcelona, Spain

${ }^{q}$ Department of Neurology and Clinical Neuroscience Center, 1st Faculty of Medicine, Charles University and General Teaching Hospital, Prague, Czech Republic

${ }^{\mathrm{r}}$ Universitätsklinikum Halle, Germany

s Tzartos NeuroDiagnostics, Athens, Greece

\section{A R T I C L E I N F O}

Article history:

Received 3 November 2014

Received in revised form 21 April 2015

Accepted 24 April 2015

\section{Keywords:}

Myasthenia gravis

MuSK

Autoantibodies

Cell based assay

Diagnosis

\begin{abstract}
A B S T R A C T
Seronegative myasthenia gravis (MG) presents a serious gap in MG diagnosis and understanding. We applied a cell based assay (CBA) for the detection of muscle specific kinase (MuSK) antibodies undetectable by radioimmunoassay. We tested 633 triple-seronegative MG patients' sera from 13 countries, detecting $13 \%$ as positive. MuSK antibodies were found, at significantly lower frequencies, in $1.9 \%$ of healthy controls and $5.1 \%$ of other neuroimmune disease patients, including multiple sclerosis and neuromyelitis optica. The clinical data of the newly diagnosed MuSK-MG patients are presented. 27\% of ocular seronegative patients were MuSK antibody positive. Moreover, $23 \%$ had thymic hyperplasia suggesting that thymic abnormalities are more common than believed.
\end{abstract}

(c) 2015 Elsevier B.V. All rights reserved.
Abbreviations: MG, myasthenia gravis; MuSK, muscle specific kinase; AChR, acetylcholine receptor; LRP4, low-density lipoprotein receptor-related protein 4; CBA, cell based assay.

* Corresponding authors at: Hellenic Pasteur Institute, 127 V. Sofias Ave., GR 11521, Athens, Greece.

E-mail addresses: zisimopoulou@pasteur.gr (P. Zisimopoulou), tzartos@pasteur.gr (S.J. Tzartos).

\section{Introduction}

Myasthenia gravis (MG) is an autoimmune disease affecting the neuromuscular junction (NMJ) of skeletal muscles, causing muscle weakness and fatigability. In up to $80 \%$ of patients this is caused by autoantibodies against the acetylcholine receptor (AChR), termed AChRMG (Meriggioli and Sanders, 2009). These autoantibodies cause destruction of the NMJ via complement, since they belong mostly to the 
IgG1 and IgG3 subclasses (Lefvert et al., 1981; Rodgaard et al., 1987), by inducing receptor antigenic modulation, or by directly blocking receptor function (Vincent and Drachman, 2002). In approximately another 6\% of MG patients autoantibodies are directed against the muscle specific kinase (MuSK), MuSK-MG; this percentage varies among populations, probably owing to geographical and genetic differences (Hoch et al., 2001; Scuderi et al., 2002; Sanders et al., 2003; Niks et al., 2007; Kostera-Pruszczyk et al., 2008; Tsiamalos et al., 2009). However, as MuSK antibodies are mostly of the IgG4 subclass, a different pathological mechanism compared to AChR-MG may be at play (McConville et al., 2004; Boneva et al., 2006; Niks et al., 2008). More recently, the lowdensity lipoprotein receptor-related protein 4 (LRP4) has been identified as a new MG autoantigen (Higuchi et al., 2011; Pevzner et al., 2012; Zhang et al., 2012). A large multinational study revealed the overall LRP4-MG rate to be around 19\% among MG patients without AChR or MuSK antibodies (Zisimopoulou et al., 2013). Moreover, it was shown that the LRP4 antibodies belong mainly to the IgG1 and IgG2 subclasses, providing further clues towards potential pathogenic mechanisms. However, there are still some patients that remain without detectable autoantibodies, termed seronegative (SN-MG); this both makes MG diagnosis difficult, and complicates the differential diagnosis.

MG is a heterogeneous disease, presenting with variable symptoms. Patients with AChR antibodies present with more pathological thymic findings than MuSK-MG. On the other hand, MuSK-MG patients have more bulbar symptoms compared to AChR-MG. Importantly different MG subgroups can have a different response to therapy. Indeed, MuSK-MG patients can present with adverse effects when treated with pyridostigmine, a cholinesterase inhibitor commonly used as a first-line treatment for MG, and there is little evidence to support the usefulness of thymectomy, while they usually greatly benefit from plasma exchange (PLEX) (Guptill et al., 2011). It is, therefore, important to be able to diagnose the patients, not only based on physical examination and electromyography data, but also serologically. The detection of the autoantigen targeted in each patient is crucial to adopt the best treatment options, and to monitor disease progression and response to therapy.

The most widely used method for autoantibody detection in MG is the radioimmunoprecipitation assay (RIPA). However, some previously seronegative patients have actually been found to have low affinity AChR antibodies, detected only when a cell based assay (CBA) was used instead (Leite et al., 2008). Furthermore, we have found that a CBA was sensitive in the detection of LRP4 antibodies (Zisimopoulou et al., 2013). CBAs are proving able to detect autoantibodies with higher sensitivity than other assays, allowing the diagnosis of previously seronegative patients and are increasingly used for routine diagnosis.

In the current study we report the use of a CBA for the sensitive detection of MuSK antibodies, in several hundred MG sera from 13 different countries. Using this CBA, MuSK antibodies were detected in $13 \%$ of previously seronegative patients. Furthermore, we provide data with respect to the clinical characteristics of the newly diagnosed patients and their response to therapy.

\section{Materials and methods}

\subsection{Sample and data collection}

The groups participating in the study contributed mainly sera from double seronegative (dSN) MG patients (i.e. without detectable AChR or MuSK antibodies), and additionally some sera from seropositive MG or non-MG individuals. Serum samples were collected from 904 MG patients [96 AChR-MG, 79 MuSK-MG, 96 LRP4-MG and 633 triple SN-MG (tSN-MG: AChR, MuSK and LRP4 antibody negative)] and 162 healthy controls (donated by personnel of the contributing laboratories, or sera acquired for routine biochemical tests) (Table 1). Additionally, sera from 128 patients with other neuroimmune diseases (OND) were tested [78 with multiple sclerosis (MS) and 50 with neuromyelitis optica (NMO)]. Based on the available information of the individuals in the control groups (about 30\%), there was no statistically significant difference between the mean age of controls and MG patient groups ( $47.9 \pm 14.4$ vs $54.4 \pm 14.5$ years old) The samples originated from 13 different countries: Norway, The Netherlands, Germany, Poland, Czech Republic, France, Italy, Serbia, Spain, Greece, Cyprus, Turkey and Israel. Sera were obtained with the informed consent of the patients. The work was approved by the Ethical Committee of the Hellenic Pasteur Institute where the CBA assays were performed. The MuSK antibody assays were performed blindly. For all but the Greek patients MG was diagnosed based on clinical features and electrophysiological findings; with the exception of Greek patients, MG patients were graded according to the MGFA classification. The patients included in the Greek cohort were selected from the Hellenic Pasteur Institute diagnostic serum biobank on the basis of the short symptom description by their doctors; therefore, these patients are not diagnosed as definitely MG, but as highly likely MG. The participating teams provided information, when available, for the samples with respect to MG severity, clinical characteristics and therapies, using a template table. Information on thymic pathology was obtained from thymectomized patients.

The distribution and severity of myasthenic weakness was classified according to the Myasthenia Gravis Foundation of America (MGFA) grading system. Initial MGFA classification was that of the first presentation. Patients with only ocular symptoms for more than 2 years since initial presentation were classified as ocular MG. Patients' response to therapy was classified according to the MGFA postintervention status (PIS).

Table 1

Serum samples contributed by each country.

\begin{tabular}{|c|c|c|c|c|c|c|c|}
\hline Country & tSN-MG & AChR-MG & MuSK-MG & LRP4-MG & $\mathrm{OND}^{\mathrm{a}}$ & Healthy & Total \\
\hline Norway & 48 & 2 & 4 & 3 & 0 & 0 & 57 \\
\hline The Netherlands & 38 & 0 & 0 & 9 & 0 & 0 & 47 \\
\hline Germany & 14 & 0 & 0 & 0 & 0 & 0 & 14 \\
\hline Poland & 44 & 29 & 5 & 16 & 0 & 0 & 94 \\
\hline Czech Republic & 27 & 0 & 0 & 3 & 0 & 0 & 30 \\
\hline France & 70 & 6 & 3 & 13 & 0 & 0 & 92 \\
\hline Italy & 49 & 28 & 28 & 13 & 30 & 25 & 173 \\
\hline Serbia & 49 & 23 & 18 & 7 & 0 & 8 & 105 \\
\hline Spain & 21 & 8 & 0 & 4 & 0 & 6 & 39 \\
\hline Greece & 96 & 0 & 15 & 2 & 98 & 123 & 334 \\
\hline Cyprus & 55 & 0 & 5 & 9 & 0 & 0 & 69 \\
\hline Turkey & 63 & 0 & 1 & 5 & 0 & 0 & 69 \\
\hline Israel & 59 & 0 & 0 & 12 & 0 & 0 & 71 \\
\hline Total & 633 & 96 & 79 & 96 & 128 & 162 & 1194 \\
\hline
\end{tabular}

a 78 MS and 50 NMO patients. 


\subsection{CBA for the detection of MuSK and AChR clusters}

CBAs were essentially performed as described by Leite et al. (2008). Briefly, 24 h prior to transfection, HEK293 cells were seeded in 96-well plate coated with $10 \%$ polyD-lysine. Cells were transiently transfected either with pCDNA3-MuSK (kindly provided by Dr. Beeson) or with the same plasmid expressing an irrelevant membrane protein (aquaporin 4 ) as a control, using polyethylenamine (Polyplus). $48 \mathrm{~h}$ post-transfection, the cells were washed three times with DMEM containing 20 mM HEPES, 10\% FBS, 1\% Pen/Strep (wash buffer) and subsequently incubated with serum samples diluted at 1:40 with wash buffer containing $1 \%$ bovine serum albumin (dilution buffer) for $1 \mathrm{~h}$ at room temperature (RT). Afterwards, cells were washed and fixed with $10 \%$ formaline solution (Sigma-Aldrich) for $15 \mathrm{~min}$ and washed again for three times. For the visualization of patients' antibodies, Alexa Fluor568 conjugated anti-human IgG antibody was used (Life Technologies, Invitrogen) diluted 1:750 in dilution buffer for $1 \mathrm{~h}$ at room temperature. This antibody reacts with the heavy chains of IgG as well as the light chains from all classes. For the determination of MuSK antibody class the same method was performed as described above (sera dilution $1: 10$ ) but the goat FITC-labeled anti-human IgG (IgG Fc specific) (Sigma-Aldrich) or the Alexa Fluor-488 conjugated anti-human IgM (IgM heavy chain specific) (Life Technologies, Invitrogen) was used as secondary antibody.

Cells were examined under an Olympus IX51 fluorescence microscope equipped with an Infinity1-2CB digital image system. Tests were performed blind. All sera were evaluated from 0: no signal, 0.5: ambiguous, 1: weak, 2: moderate, 3: strong and 4: very strong signal by two observers in three independent experiments. The score (between observers) did not vary in the case of 0 , whereas there was a variation of $\leq 1$ point in the other sera. Sera with average ambiguous score (0.5) were considered as negative.

The CBA for the detection of AChR clusters was performed as described above using the plasmids provided by Dr Beeson encoding for $\alpha, \beta, \gamma, \delta$, and $\varepsilon$ AChR subunits and rapsyn transfected in a ratio of $2: 1: 1: 1: 1: 1$, respectively.

\subsection{Radioimmunoprecipitation assays (RIPA) for AChR and MuSK antibodies}

AChR and MuSK antibody seropositivity and titers were determined using the commercial AChR and MuSK antibody RIPA kits (RSR Ltd, Cardiff, UK) according to the manufacturer's instructions. AChR antibody titers lower than $0.2 \mathrm{nM}$ or higher than $0.5 \mathrm{nM}$ were considered negative or positive, respectively, whereas values between $0.2 \mathrm{nM}$ and $0.5 \mathrm{nM}$ were considered ambiguous. Similarly, MuSK antibody titers lower than $0.02 \mathrm{nM}$ or higher than $0.05 \mathrm{nM}$ were considered negative or positive, respectively, whereas values between 0.02 and $0.05 \mathrm{nM}$ were considered ambiguous. All ambiguous sera were excluded from this study. The seropositivity of the samples was provided by the different originating clinics, but all sera found MuSK-CBA positive were retested for MuSK antibodies by RIPA at the Hellenic Pasteur Institute.

\subsection{Statistical analysis}

Statistical analyses were performed using the Student's $t$ test or Yates' corrected chi-square test.

\section{Results}

\subsection{Detection of MuSK antibodies with CBA}

A CBA was used for the reliable and efficient detection of MuSK antibodies. Initially, sera from 20 MuSK-MG patients (determined by RIPA) as well as from 20 healthy donors were used in order to optimize the assay's conditions. The healthy control sera at a dilution of 1:40 and under the conditions described in the Materials and methods section gave no signal, whereas all MuSK antibody positive sera (by RIPA, including the low titer ones) received a score higher than 2 (Fig. 1).

To evaluate further the assay in terms of specificity, an additional 142 healthy control sera and 128 sera from patients with other neuroimmune diseases ( 78 of which had MS and 50 NMO) were tested. 3 of the healthy controls and 7 of the OND patients' samples tested positive (Table 2).

Using this CBA, we tested MG patient sera from 13 countries (Norway, the Netherlands, Germany, Poland, the Czech Republic, France, Italy, Serbia, Spain, Greece, Cyprus, Turkey and Israel). All patients that were known MuSK-MG (79 samples) by means of the MuSK antibody RIPA were also detected as positives with the CBA. In addition, the CBA detected MuSK antibodies in 13.1\% of the 633 tSN-MG sera tested (those without previously detected AChR, MuSK or LRP4 antibodies). There was considerable variability among the different populations involved in the study, with seropositivity against MuSK by CBA ranging from $4.8 \%$ in the Spanish cohort to $22.4 \%$ in the Serbian cohort (Fig. 2A). MuSK seropositivity by CBA did not correlate with the geographical location (latitude) (Fig. 2B). The screening of known AChRMG and LRP4-MG sera revealed the presence of MuSK antibodies in a number of these, i.e. several patients were double positive for more than one antigen; $12.5 \%$ (12 of 96 AChR-MG sera tested) were AChR/ MuSK double positives and 19.8\% (19/96) LRP4/MuSK double positives (Table 2). There were 2 samples that tested positive for all three antigens, both originating from the Serbian cohort.

The average age at disease onset of patients with CBA-detected MuSK antibodies was 34 years, with the majority of patients presenting before the age of 40 (71.5\%) and only $17 \%$ above the age of 50 (Fig. 3 ). Interestingly, the mean age of the seronegative patients who were negative in the MuSK CBA was 40 years. The age distribution among females and males differed significantly ( $p<0.01$ ), with the former being prevalent in younger ages (mean onset age $=31$ ) and the later in older (mean onset age $=40$ ). The majority of patients were female, although not as prominently as reported for other MG groups, with a female/male ratio of $1.5 / 1$.

Looking for differences in the nature of MuSK antibodies identified only with the CBA and those identified with both CBA and RIPA we tested sera belonging to both categories (25 CBA positive only and 5 CBA and RIPA positive) with a CBA assay using fluorescence conjugated anti-human IgM and IgG (FC specific) as a secondary antibody. We
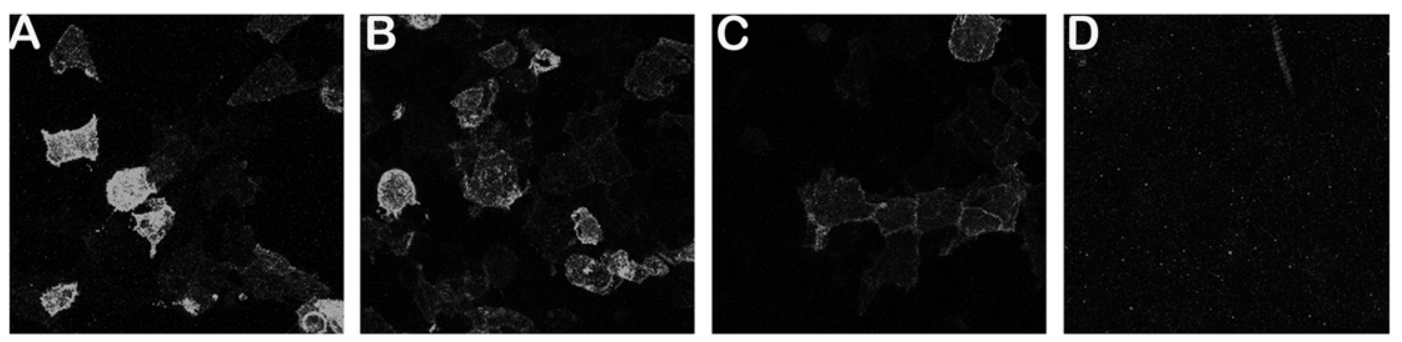

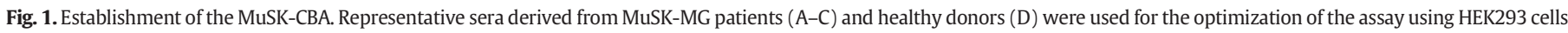
expressing human MuSK. A: very strong signal/score 4; B: strong signal/score 3; C: weak signal/score 1; D: no signal/score 0 (healthy donor). 
Table 2

Anti-MuSK positive sera with the CBA in the various patients' cohorts.

\begin{tabular}{lcll}
\hline Sera & Tested & MuSK CBA + & Percentage \\
\hline tSN-MG & 633 & 83 & $13.1 \%$ \\
AChR-MG (by RIPA) & 96 & 12 & $12.5 \%$ \\
MuSK-MG (by RIPA) & 79 & 79 & $100 \%$ \\
LRP4-MG (by CBA) & 96 & 19 & $19.8 \%$ \\
OND & 128 & 7 & $5.1 \%(\mathrm{p}<0.01)$ \\
Healthy & 162 & 3 & $1.9 \%(\mathrm{p}<0.001)$ \\
\hline
\end{tabular}

found that all 25 sera only positive with the CBA contained IgM MuSK antibodies (identified with the IgM specific antibody), whereas only 2/ 25 contained also IgG MuSK antibodies (identified with anti-IgG Fc specific antibody) (data not shown). In contrast, MuSK antibodies from RIPA positive sera, as expected, belonged mainly to the IgG class with 2/5 sera also having IgM MuSK antibodies.

180 randomly chosen MG samples remaining seronegative after the MuSK CBA screening were also tested by CBA for autoantibodies against clustered AChRs, as previously described (Leite et al., 2008). Of these, 7 (4\%) were found positive.

\subsection{Clinical presentation of patients positive in MuSK-CBA only}

The clinical characteristics of the newly identified patients were collected and analyzed to detect possible unique features. Overall, these patients presented with mild disease at onset, since the majority (77.1\%) were scored with MGFA grade I or II at first diagnosis, 22.9\% with moderate to severe (MGFA grades III and IV), and none received a grade of $\mathrm{V}$ (Table 3 ). This resembles the distribution among the triple seronegative and LRP4-MG groups, but is significantly milder than patients with AChR-MG ( $\mathrm{p}<0.05)$. Only 2 out of 43 CBA-positive MuSKMG patients with available data suffered a myasthenic crisis at some point. Interestingly, $18.7 \%$ of CBA-positive MuSK-MG remained purely ocular for more than two years (Table 3 ). These actually account for $15.8 \%$ of previously seronegative ocular MG patients and their distribution among the different countries is summarized in Table 4. These observations are not due to the detection of antibodies in patients recently diagnosed, since the mean time from diagnosis to sample collection for this study was 14.7 years for the CBA positive sera and 8.7 years for the RIPA positive ones, i.e. they were patients remaining seronegative for many years.

The presentation of symptoms with respect to affected muscles was almost equally divided since in $44.8 \%$ of patients limb or axial muscles were primarily affected, while in $55.2 \%$ there was bulbar or respiratory muscle involvement. Moreover, 7\% of CBA-positive MuSK-MG patients developed muscle atrophy (facial and pharyngeal muscle atrophy reported).

Thymic abnormalities are often observed in MG patients, so we collected information with respect to thymic pathologies among these patients, where available. Although more than half of the patients had
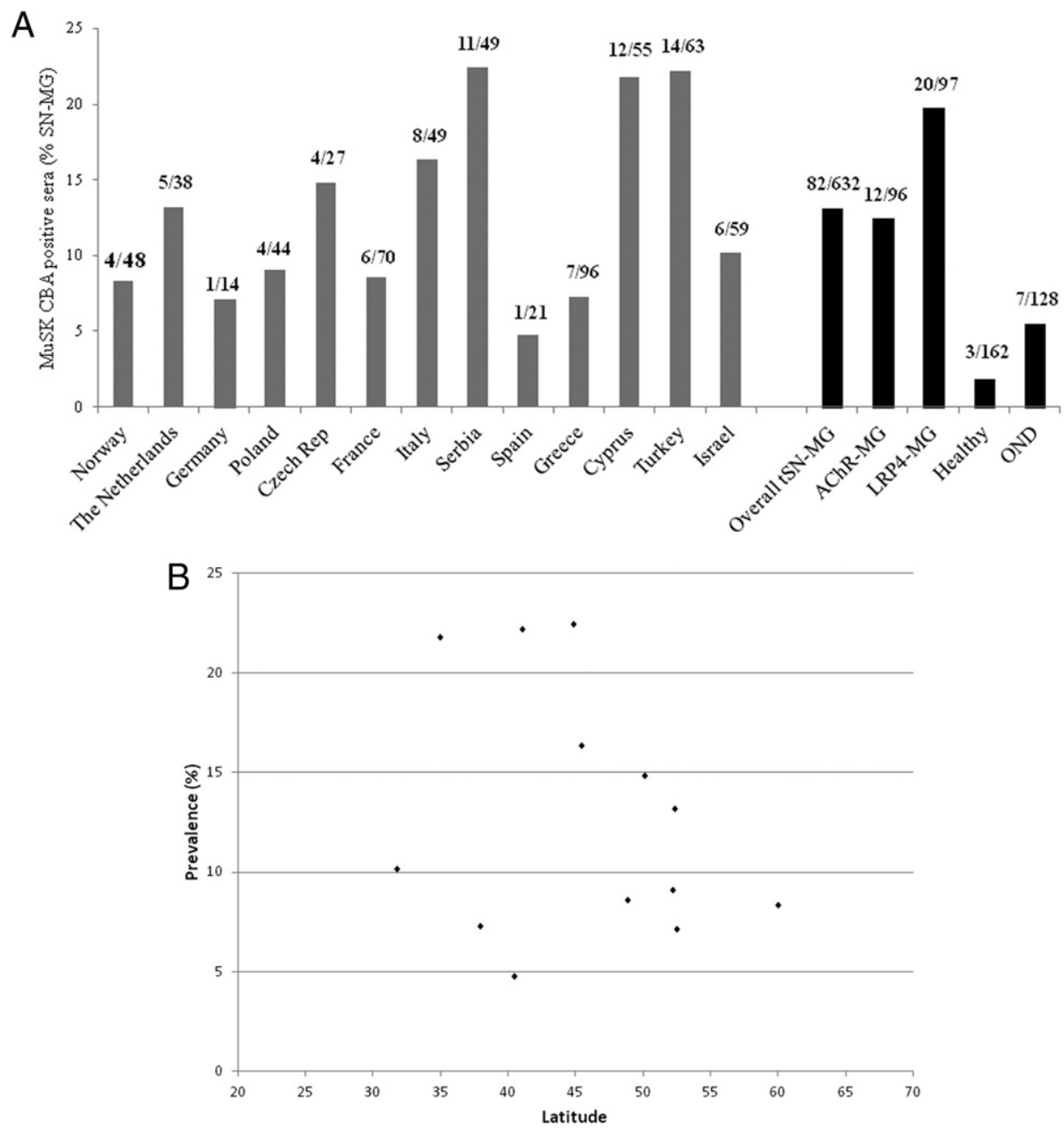

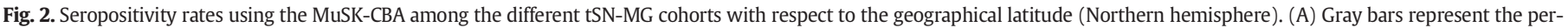

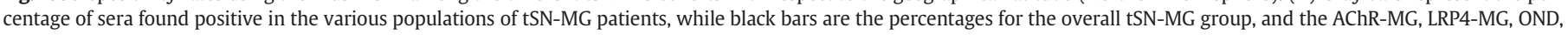
and healthy control groups. (B) The distribution of anti-MuSK positives using the CBA does not correlate with the geographic origin of the cohorts. 


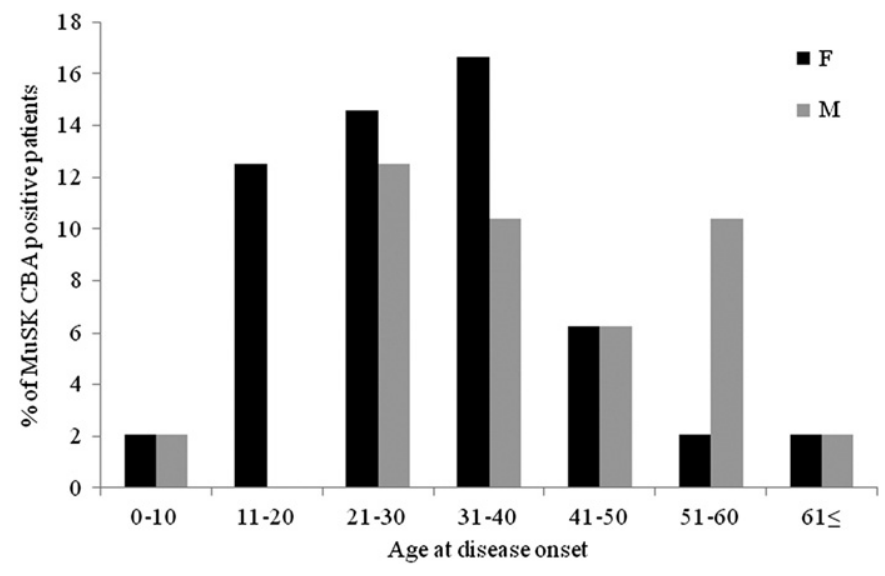

Fig. 3. Age distribution of disease onset among the LRP4-MG patients $(\mathrm{N}=48)$.

normal for age thymi, hyperplasia was found in $23 \%$ of cases and atrophy or involuted thymus in $20 \%$; no cases of thymoma were reported (Table 5).

\subsection{Response to therapy}

The various treatment regimes adopted by each patient were analyzed, when available. This was done retrospectively, since these patients were seronegative at the time of treatment. The data revealed that most patients received the acetylcholinesterase inhibitor (AChE-I) pyridostigmine, a first line drug in the management of MG. Only half of the treated patients had a good response, while approximately $10 \%$ did not respond at all (Table 6). The second most common medication was the frequently used corticosteroid prednisone, used in $52 \%$ of patients, all of which had a good ( $81 \%$ ) or partial (19\%) response. Additional treatments included azathioprine (27\% of patients treated), IVIg and PLEX ( $18 \%$ and $15 \%$ of patients respectively). The last two treatments were often performed in the same patients, so that overall, $23.5 \%$ of the patients were treated with at least one of these acute treatments, and all of them benefitted from the treatment. Finally, 22.5\% of patients underwent thymectomy. These were mostly women (78\%) with early onset (mean age $=27$ years old), but limited information and the variety of concomitant treatments did not allow the evaluation of its therapeutic benefit.

Overall, $26 \%$ of patients positive in the MuSK CBA went into remission (7.5\% complete stable and $18.5 \%$ pharmacological remission), and a further $59 \%$ showed minimal manifestations or improvement of their clinical status, while there were no cases with reported worsening of their post-intervention status (Table 6). There were no MG-related reported deaths.

Table 3

Clinical data summary of MG patients.

\begin{tabular}{llccccr}
\hline MG subgroup & & MuSK-CBA $^{\mathrm{b}}$ & MuSK-RIPA & AChR-RIPA & LRP4-CBA & SN-MG $^{\mathrm{c}}$ \\
\hline MGFA (at first & I & $14 / 48$ & $3 / 25$ & $7 / 51$ & $20 / 67$ & $80 / 244$ \\
diagnosis) & II & $23 / 48$ & $10 / 25$ & $19 / 51$ & $37 / 67$ & $122 / 244$ \\
& III & $10 / 48$ & $11 / 25$ & $15 / 51$ & $8 / 67$ & $33 / 244$ \\
& IV & $1 / 48$ & $1 / 25$ & $9 / 51$ & $2 / 67$ & $5 / 244$ \\
& V & $0 / 48$ & $0 / 25$ & $1 / 51$ & $0 / 67$ & $4 / 244$ \\
Ocular >2 years & $9 / 48$ & $1 / 7$ & $4 / 28$ & $15 / 67$ & $48 / 244$ \\
Bulbar & $13 / 29$ & $17 / 22$ & $33 / 44$ & $25 / 38$ & $83 / 151$ \\
predominance & & & & & \\
Myasthenic crises & $2 / 43$ & $1 / 22$ & $8 / 43$ & $4 / 61$ & $13 / 200$ \\
Muscle atrophy & $2 / 41$ & $1 / 22$ & $1 / 52$ & $5 / 61$ & $3 / 180$ \\
\hline
\end{tabular}

a Ratios represent the number of patients with a specific characteristic over the total

number of patients with available data for that category.

b Patients only positive by the MuSK-CBA and negative by the RIPA.

c Patients still seronegative by all RIPA and CBA assays against all 3 antigens.
Table 4

Prevalence of CBA MuSK positive patients with ocular MG.

\begin{tabular}{lll}
\hline \multirow{2}{*}{ Country } & \multicolumn{2}{l}{ Ocular MG } \\
\cline { 2 - 3 } & SN-MG & MuSK-CBA \\
\hline Poland & 12 & 0 \\
Czech Rep & 4 & $1(25 \%)$ \\
Serbia & 12 & $2(17 \%)$ \\
Spain & 12 & $1(8 \%)$ \\
Turkey & 17 & $5(29 \%)$ \\
Total patients & 57 & $9(15.8 \%)$ \\
\hline
\end{tabular}

a Patients still seronegative by all RIPA and CBA assays against all 3 antigens.

\section{Discussion}

Until recently, for MG diagnosis, RIPA was the gold standard in autoantibody detection, though ELISAs were also commercially available. The former, allowed the detection of $80-85 \%$ of patients having AChR antibodies, and a further $\sim 6 \%$ with MuSK antibodies. The remaining seronegative patients present a gap in the diagnosis of MG and the differential diagnosis of related disorders. In addition to the quest for new autoantigen targets, the improvement of the assay sensitivity was thought to help minimize the number of seronegative patients. Attempts to improve the sensitivity of the RIPA resulted in a two-step assay with lower detection limits (Trakas et al., 2011). Still, this did not increase significantly the group of MuSK-MG patients (Trakas and Tzartos, unpublished results).

CBAs are increasingly used in routine diagnosis for the detection of MG autoantibodies as they seem able to detect antibodies in sera found seronegative with the conventional RIPA and ELISA methods (Leite et al., 2010; Vincent et al., 2012; Devic et al., 2014). We used in principle the CBA developed by Leite et al. (2008) for the detection of antibodies against MuSK, using HEK293 cells expressing the fulllength human MuSK protein. MS and NMO were specifically selected since they are neuroimmune disorders providing a stringent control for the possibility of non-disease specific antibodies. The higher frequency of MG among MS and NMO patients could explain the higher percentage of positives in the OND than the healthy control group. At this point we do not know whether these antibodies predispose for disease, likely requiring additional factors for disease to develop. The pathogenic significance of autoantibodies detected by the MuSK CBA in MG and other neuroimmune diseases remains to be fully investigated. To keep the non-specific binding to a minimum, the sera were diluted 1:40; this unavoidably resulted in a decrease in the number of patients detected as seropositive as well. With these conditions, the specificity of the CBA was above $98 \%$, comparable to the $97 \%$ specificity rate recently reported for similar assays (Rodriguez Cruz et al., 2015). Since this assay is not $100 \%$ specific for MG, it requires further optimization before it can be used as a first line diagnostic of unselected patients. However, it may be used in patients with suspected MG, but a seronegative status by currently used MG assays, helping in their definite diagnosis.

Using the above CBA, we proceeded to perform a screening of MG patient sera from several European centers, mostly tSN-MG patient samples (633 tSN-MG sera from 13 countries). By collecting and analyzing the clinical data of these patients, we also sought to investigate the clinical characteristics of the MuSK-MG patients detected by the CBA,

Table 5

Thymic pathology.

\begin{tabular}{llllll}
\hline & MuSK-CBA & MuSK-RIPA & AChR-RIPA & LRP4-CBA & SN-MG $^{\text {a }}$ \\
\hline Total patients & 30 & 19 & 18 & 42 & 146 \\
Normal & $17(57 \%)$ & $1(5 \%)$ & 0 & $14(33 \%)$ & $76(52 \%)$ \\
Hyperplasia & $7(23 \%)$ & $4(21 \%)$ & $12(67 \%)$ & $13(31 \%)$ & $27(19 \%)$ \\
Atrophy/involuted & $6(20 \%)$ & $14(74 \%)$ & $5(28 \%)$ & $15(36 \%)$ & $38(26 \%)$ \\
Thymoma & 0 & 0 & $1(5 \%)$ & 0 & $5(3 \%)$ \\
\hline a Patients still seronegative by all RIPA and CBA assays against all 3 antigens.
\end{tabular}


Table 6

Summary of applied therapies.

\begin{tabular}{|c|c|c|c|c|}
\hline MG subgroup & & MuSK-CBA & LRP4-CBA & $\mathrm{SN}-\mathrm{MG}^{\mathrm{a}}$ \\
\hline \multirow[t]{4}{*}{ Pyridostigmine } & Treated/total ${ }^{\mathrm{b}}$ & $42 / 42$ & $42 / 42$ & $126 / 126$ \\
\hline & Response Good & 22 & 31 & 106 \\
\hline & Moderate & 16 & 8 & 10 \\
\hline & None & 4 & 3 & 10 \\
\hline \multirow[t]{4}{*}{ Prednisone } & Treated/total & $21 / 41$ & $31 / 41$ & $88 / 119$ \\
\hline & Response Good & 17 & 22 & 77 \\
\hline & Moderate & 4 & 6 & 8 \\
\hline & None & 0 & 3 & 3 \\
\hline Azathioprine & Treated/total & $10 / 37$ & $11 / 27$ & $43 / 137$ \\
\hline \multirow[t]{7}{*}{ Overall response (PIS) } & Total & 27 & 46 & 140 \\
\hline & $\mathrm{CSR}^{\mathrm{c}}$ & 2 & 3 & 19 \\
\hline & $P^{d}$ & 5 & 11 & 25 \\
\hline & $\mathrm{MM}^{\mathrm{e}}$ & 6 & 7 & 34 \\
\hline & Improved & 10 & 16 & 42 \\
\hline & Unchanged & 4 & 8 & 17 \\
\hline & Worse & 0 & 1 & 3 \\
\hline
\end{tabular}

a Patients still seronegative by all RIPA and CBA assays against all 3 antigens.

b Number of patients receiving the specific therapy vs. total patients with available clinical information for each category.

c $\mathrm{CSR}=$ complete stable remission

d $\mathrm{PR}=$ pharmacologic remission.

e $\mathrm{MM}=$ minimal manifestations.

identifying similarities and differences with typical MuSK-MG and the other MG subtypes.

In terms of prevalence, there was considerable variation among the different geographical populations examined (from 4.8 to $22 \%$ ), suggesting the contribution of environmental and/or genetic factors in the pathogenesis of the disease. A geographical pattern has been described for MuSK-MG detected by RIPA, whereby there is a higher prevalence closer to the equator and smaller towards the poles (Vincent et al., 2008), but we could not detect such a correlation here. MuSKMG by RIPA is also less prevalent in Japanese populations (Suzuki et al., 2011), but none of our cohorts included Asian patients for comparison. It would be interesting to investigate, using the more sensitive $\mathrm{CBA}$, whether the lower seropositivity is due to the non-detection of the MuSK antibodies by conventional assays, or indeed prevalence is lower in the Japanese population.

Though typically MuSK antibodies detected by RIPA belong mainly to the IgG4 subclass, the MuSK antibodies detected with the CBA were mostly non-IgG and contained antibodies of the IgM class. This observation is in agreement with previous publications suggesting that IgM antibodies were responsible for MG symptoms in some SN-MG patients (Yamamoto et al., 1991; Plested et al., 2002; Vincent et al., 2003). The IgM in those studies were not identified as MuSK antibodies, probably due to the low affinity of the IgM antibodies and therefore their inability to bind on the soluble protein used in RIPA experiments.

When sera positive for AChR or LRP4 antibodies were screened with the MuSK CBA, a relatively high prevalence of double positives, i.e. AChR or LRP4 antibody positive sera that also had MuSK antibodies, was found (12.5 and $19.8 \%$ respectively). Previously, using a CBA for the detection of LRP4 antibodies, 15\% MuSK/LRP4 double positives had been reported (Zisimopoulou et al., 2013). The considerable overlap in autoantigen repertoire could be explained by the close proximity and interaction of the MuSK and LRP4 proteins at the NMJ, with the possibility of some antibodies recognizing the MuSK-LRP4 interfaces. In fact, MuSK antibodies have been shown to act, at least in part, by preventing the MuSK-LRP4 interaction (Huijbers et al., 2013; Koneczny et al., 2013), suggesting that they target epitopes close to or at the interacting regions. On the other hand, MuSK/AChR double positives have rarely been reported (Ohta et al., 2004; Poulas et al., 2012; Zouvelou et al., 2013). However, the MuSK CBA detected a higher than expected rate of AChR-MG patients with concomitant presence of MuSK antibodies.

The age distribution of disease onset showed a characteristic pattern. In AChR-MG there are two peaks of incidence, one around the third decade of life with female predominance and a second around the sixth decade with male predominance (Poulas et al., 2001). The patients found positive by the MuSK CBA developed symptoms around the fourth decade of life, following a distribution similar to that of typical MuSK-MG (Guptill et al., 2011). MuSK antibodies were found more commonly in women (F/M ratio 1.5/1), albeit much less prominently than the 3.6-4/1 female/male ratios reported for MuSK-MG by RIPA (Evoli et al., 2003; Vincent et al., 2008). Furthermore, symptoms developed at a younger age in women than in men, comparable to other MG subtypes.

In ocular MG patients, only about $50 \%$ have detectable AChR antibodies (Meriggioli and Sanders, 2012). The remaining were considered seronegative (McConville et al., 2004), with the exception of a few cases of well documented purely ocular MuSK-MG (Caress et al., 2005; Bau et al., 2006; Chan and Orrison, 2007; Yang et al., 2011). Recently, we had shown that up to $27 \%$ of the ocular SN-MG patients were LRP4 antibody positive (Zisimopoulou et al., 2013). Here we showed that an additional $15.8 \%$ of the seronegative patients with ocular MG are actually MuSK-MG, suggesting that MuSK-MG is much more common among ocular MG than previously thought.

The great majority of MuSK-CBA positive patients (77\%) had an MGFA classification at onset of I or II, resembling the distribution of the SN-MG and LRP4-MG patients. Due to limited information with respect to the maximum severity of symptoms it remains uncertain whether these patients have an overall milder disease manifestation (Baggi et al., 2013). Nevertheless, much fewer of the MuSK-CBA positive patients (4.6\%) presented with myasthenic crises compared to the 2548\% reported for typical AChR-MG and MuSK-MG (Oh, 2009; Evoli and Padua, 2013). A correlation of antibody levels and disease severity has been suggested in previous studies of MuSK-MG patients (Bartoccioni et al., 2006). Our results could support this observation as the CBA might detect patients with lower anti-MuSK levels, which accordingly might have milder disease. However, since the majority of sera detected only by the CBA contain MuSK antibodies belonging to the IgM class but not to the IgG class, the difference with the MuSK $\mathrm{CBA}$ is probably more qualitative rather than quantitative.

The thymus has been implicated in the pathogenesis of MG and thymic pathologies (e.g. hyperplasia, thymoma) are well known (Marx et al., 2013). Specifically, thymic hyperplasia is often found in AChRMG and to a lesser extent in SN-MG, which on the other hand is thought to be rare in the case of MuSK-MG (Lauriola et al., 2005; Leite et al., 2005). Additionally, thymoma is found in up to $15 \%$ of AChR-MG, but in MuSK-MG a coexisting thymoma is a rare finding (Meriggioli and Sanders, 2009; Yang et al., 2011). In agreement with these observations, we did not find any thymoma cases in patients positive with the MuSKCBA. Furthermore, the effectiveness of thymectomy is questioned in MuSK-MG (Evoli et al., 2008), while thymic involvement in its pathogenesis is uncertain. However our results show a considerable $23 \%$ of CBA positive patients with thymic hyperplasia suggesting that thymic alterations may be more common in MuSK-MG than currently assumed (Baggi et al., 2013).

The management of MG can be difficult, especially due to the variable responses of the different MG subgroups. Administration of pyridostigmine, a common AChE-I, in MuSK-MG patients can have a limited effect, or even lead to intolerance and worsening of the clinical condition (Evoli et al., 2003; Hatanaka et al., 2005; Evoli and Padua, 2013). Indeed, only half of the recorded patients in our CBA MuSK-MG cohort had a positive reaction to pyridostigmine, while the rest responded poorly or not at all, as expected for typical MuSK-MG. Overall, MuSK-CBA positive patients responded well to treatment, since none showed worsening after therapy initiation and 19\% did not show improvement. Nevertheless, recurring symptoms were present and the majority of the patients received a combination immunosuppressive regime for a satisfactory outcome.

In order to obtain the complete picture with respect to autoantigen repertoire in our cohort, we tested some of the still seronegative 
samples with a CBA for clustered AChRs. Previous reports have suggested that a fraction of the seronegative patients might have antibodies against clustered AChRs detectable by a CBA, ranging from $16 \%$ to $66 \%$ (Leite et al., 2008; Jacob et al., 2012; Chang et al., 2014; Devic et al., 2014). In our cohort, we were only able to detect approximately $4 \%$ of the samples as positive. Overall, in this cohort of patients (seronegative by RIPA for AChR and MuSK antibodies), and using CBAs for the detection of LRP4, MuSK and clustered AChR antibodies, we were able to identify $32 \%$ of patients seronegative by RIPA as positive for one or more of these autoantigens.

In conclusion, the use of a CBA with the full-length human MuSK for the screening of a large number of MG sera from 13 countries was able to detect previously seronegative patients as MuSK-MG. The frequency of patients positive with the CBA was highly variable between populations, though no geographical pattern was discerned. The identification of MuSK antibodies by the CBA in some healthy controls $(1.2 \%)$ and some patients with OND (5.1\%) should be taken into account for the final diagnosis of MG by the clinicians. Overall, the clinical presentation was milder than the typical AChR-MG and MuSK-MG subgroups, whereas the response to therapy was satisfactory. Interestingly, we also detected a significant number of double positives (AChR/MuSK-MG, LRP4/MuSK-MG, and in a previous study AChR/LRP4-MG), suggesting that their overall rate is more frequent than previously thought. Further investigation of such cases could help illuminate the pathophysiology of MG, and the underlying triggering mechanisms.

\section{Acknowledgments}

This work was supported by grants from the European Co (FP7 Fight-MG, Contract no. 242210), the Greek NSRF ("Thalis" no. 380221, "Aristeia" no. 1154 and "Kripis" no. 450598), the Association Francaise contre les Myopathies (AFM, no. 15861), the Muscular Dystrophy Association of the USA (MDA, 255236), the Polish-Norwegian Research Fund Grant PNRF 204-AI-1/07, the Grant Agency of Charles University (no. 351011/2011) and several national MG associations, including the Cyprus and Greek MG Associations, for their assistance in sera collection.

\section{References}

Baggi, F., Andreetta, F., Maggi, L., Confalonieri, P., Morandi, L., Salerno, F., et al., 2013. Complete stable remission and autoantibody specificity in myasthenia gravis. Neurology 80, 188-195.

Bartoccioni, E., Scuderi, F., Minicuci, G.M., Marino, M., Ciaraffa, F., Evoli, A., 2006. AntiMuSK antibodies: correlation with myasthenia gravis severity. Neurology 67, 505-507.

Bau, V., Hanisch, F., Hain, B., Zierz, S., 2006. Ocular involvement in MuSK antibody-positive myasthenia gravis. Klin. Monatsbl. Augenheilkd. 223, 81-83.

Boneva, N., Hamra-Amitay, Y., Wirguin, I., Brenner, T., 2006. Stimulated-single fiber electromyography monitoring of anti-sense induced changes in experimental autoimmune myasthenia gravis. Neurosci. Res. 55, 40-44.

Caress, J.B., Hunt, C.H., Batish, S.D., 2005. Anti-MuSK myasthenia gravis presenting with purely ocular findings. Arch. Neurol. 62, 1002-1003.

Chan, J.W., Orrison, W.W., 2007. Ocular myasthenia: a rare presentation with MuSK antibody and bilateral extraocular muscle atrophy. Br. J. Ophthalmol. 91, 842-843.

Chang, T., Leite, M.I., Senanayake, S., Gunaratne, P.S., Gamage, R., Riffsy, M.T., et al., 2014. Clinical and serological study of myasthenia gravis using both radioimmunoprecipitation and cell-based assays in a South Asian population. J. Neurol. Sci. 343, 82-87.

Devic, P., Petiot, P., Simonet, T., Stojkovic, T., Delmont, E., Franques, J., et al., 2014. Antibodies to clustered acetylcholine receptor: expanding the phenotype. Eur. J. Neurol. 21, 130-134.

Evoli, A., Padua, L., 2013. Diagnosis and therapy of myasthenia gravis with antibodies to muscle-specific kinase. Autoimmun. Rev. 12, 931-935.

Evoli, A., Tonali, P.A., Padua, L., Monaco, M.L., Scuderi, F., Batocchi, A.P., et al., 2003. Clinical correlates with anti-MuSK antibodies in generalized seronegative myasthenia gravis. Brain 126, 2304-2311.

Evoli, A., Bianchi, M.R., Riso, R., Minicuci, G.M., Batocchi, A.P., Servidei, S., et al., 2008. Response to therapy in myasthenia gravis with anti-MuSK antibodies. Ann. N. Y. Acad. Sci. 1132, 76-83.

Guptill, J.T., Sanders, D.B., Evoli, A., 2011. Anti-MuSK antibody myasthenia gravis: clinical findings and response to treatment in two large cohorts. Muscle Nerve 44, 36-40.
Hatanaka, Y., Hemmi, S., Morgan, M.B., Scheufele, M.L., Claussen, G.C., Wolfe, G.I., et al, 2005. Nonresponsiveness to anticholinesterase agents in patients with MuSKantibody-positive MG. Neurology 65, 1508-1509.

Higuchi, O., Hamuro, J., Motomura, M., Yamanashi, Y., 2011. Autoantibodies to lowdensity lipoprotein receptor-related protein 4 in myasthenia gravis. Ann. Neurol. 69, 418-422.

Hoch, W., McConville, J., Helms, S., Newsom-Davis, J., Melms, A., Vincent, A., 2001. Autoantibodies to the receptor tyrosine kinase MuSK in patients with myasthenia gravis without acetylcholine receptor antibodies. Nat. Med. 7, 365-368.

Huijbers, M.G., Zhang, W., Klooster, R., Niks, E.H., Friese, M.B., Straasheijm, K.R., et al., 2013. MuSK IgG4 autoantibodies cause myasthenia gravis by inhibiting binding between MuSK and Lrp4. Proc. Natl. Acad. Sci. U. S. A. 110, 20783-20788.

Jacob, S., Viegas, S., Leite, M.I., Webster, R., Cossins, J., Kennett, R., et al., 2012. Presence and pathogenic relevance of antibodies to clustered acetylcholine receptor in ocular and generalized myasthenia gravis. Arch. Neurol. 69, 994-1001.

Koneczny, I., Cossins, J., Waters, P., Beeson, D., Vincent, A., 2013. MuSK myasthenia gravis IgG4 disrupts the interaction of LRP4 with MuSK but both IgG4 and IgG1-3 can disperse preformed agrin-independent AChR clusters. PLoS One 8, e80695.

Kostera-Pruszczyk, A., Kaminska, A., Dutkiewicz, M., Emeryk-Szajewska, B., StrugalskaCynowska, M.H., Vincent, A., et al., 2008. MuSK-positive myasthenia gravis is rare in the Polish population. Eur. J. Neurol. 15, 720-724.

Lauriola, L., Ranelletti, F., Maggiano, N., Guerriero, M., Punzi, C., Marsili, F., et al., 2005. Thymus changes in anti-MuSK-positive and -negative myasthenia gravis. Neurology 64 $536-538$.

Lefvert, A.K., Cuenoud, S., Fulpius, B.W., 1981. Binding properties and subclass distribution of anti-acetylcholine receptor antibodies in myasthenia gravis. J. Neuroimmunol. 1, $125-135$.

Leite, M.I., Strobel, P., Jones, M., Micklem, K., Moritz, R., Gold, R., et al., 2005. Fewer thymic changes in MuSK antibody-positive than in MuSK antibody-negative MG. Ann. Neurol. 57, 444-448.

Leite, M.I., Jacob, S., Viegas, S., Cossins, J., Clover, L., Morgan, B.P., et al., 2008. IgG1 antibodies to acetylcholine receptors in 'seronegative' myasthenia gravis. Brain 131, 1940-1952.

Leite, M.I., Waters, P., Vincent, A., 2010. Diagnostic use of autoantibodies in myasthenia gravis. Autoimmunity 43, 371-379.

Marx, A., Pfister, F., Schalke, B., Saruhan-Direskeneli, G., Melms, A., Strobel, P., 2013. The different roles of the thymus in the pathogenesis of the various myasthenia gravis subtypes. Autoimmun. Rev. 12, 875-884.

McConville, J., Farrugia, M.E., Beeson, D., Kishore, U., Metcalfe, R., Newsom-Davis, J., et al, 2004. Detection and characterization of MuSK antibodies in seronegative myasthenia gravis. Ann. Neurol. 55, 580-584.

Meriggioli, M.N., Sanders, D.B., 2009. Autoimmune myasthenia gravis: emerging clinica and biological heterogeneity. Lancet Neurol. 8, 475-490.

Meriggioli, M.N., Sanders, D.B., 2012. Muscle autoantibodies in myasthenia gravis: beyond diagnosis? Expert. Rev. Clin. Immunol. 8, 427-438.

Niks, E.H., Kuks, J.B., Verschuuren, J.J., 2007. Epidemiology of myasthenia gravis with antimuscle specific kinase antibodies in The Netherlands. J. Neurol. Neurosurg. Psychiatry 78, 417-418.

Niks, E.H., van Leeuwen, Y., Leite, M.I., Dekker, F.W., Wintzen, A.R., Wirtz, P.W., et al., 2008 Clinical fluctuations in MuSK myasthenia gravis are related to antigen-specific IgG4 instead of IgG1. J. Neuroimmunol. 195, 151-156.

Oh, S.J., 2009. Muscle-specific receptor tyrosine kinase antibody positive myasthenia gravis current status. J. Clin. Neurol. 5, 53-64.

Ohta, K., Shigemoto, K., Kubo, S., Maruyama, N., Abe, Y., Ueda, N., et al., 2004. MuSK antibodies in AChR Ab-seropositive MG vs AChR Ab-seronegative MG. Neurology 62 2132-2133.

Pevzner, A., Schoser, B., Peters, K., Cosma, N.C., Karakatsani, A., Schalke, B., et al., 2012. Anti-LRP4 autoantibodies in AChR- and MuSK-antibody-negative myasthenia gravis J. Neurol. 259, 427-435.

Plested, C.P., Tang, T., Spreadbury, I., Littleton, E.T., Kishore, U., Vincent, A., 2002. AChR phosphorylation and indirect inhibition of AChR function in seronegative MG. Neurology 59, 1682-1688.

Poulas, K., Tsibri, E., Kokla, A., Papanastasiou, D., Tsouloufis, T., Marinou, M., et al., 2001. Epidemiology of seropositive myasthenia gravis in Greece. J. Neurol. Neurosurg. Psychiatry $71,352-356$.

Poulas, K., Koutsouraki, E., Kordas, G., Kokla, A., Tzartos, S.J., 2012. Anti-MuSK- and antiAChR-positive myasthenia gravis induced by d-penicillamine. J. Neuroimmunol. 250, 94-98.

Rodgaard, A., Nielsen, F.C., Djurup, R., Somnier, F., Gammeltoft, S., 1987. Acetylcholine receptor antibody in myasthenia gravis: predominance of IgG subclasses 1 and 3. Clin. Exp. Immunol. 67, 82-88.

Rodriguez Cruz, P.M., Huda, S., Lopez-Ruiz, P., Vincent, A., 2015. Use of cell-based assays in myasthenia gravis and other antibody-mediated diseases. Exp. Neurol. http://dx.doi. org/10.1016/j.expneurol.2015.01.011.

Sanders, D.B., El-Salem, K., Massey, J.M., McConville, J., Vincent, A., 2003. Clinical aspects of MuSK antibody positive seronegative MG. Neurology 60, 1978-1980.

Scuderi, F., Marino, M., Colonna, L., Mannella, F., Evoli, A., Provenzano, C., et al., 2002. Antip110 autoantibodies identify a subtype of "seronegative" myasthenia gravis with prominent oculobulbar involvement. Lab. Investig. 82, 1139-1146.

Suzuki, S., Utsugisawa, K., Nagane, Y., Satoh, T., Kuwana, M., Suzuki, N., 2011. Clinical and immunological differences between early and late-onset myasthenia gravis in Japan. J. Neuroimmunol. 230, 148-152.

Trakas, N., Zisimopoulou, P., Tzartos, S.J., 2011. Development of a highly sensitive diagnostic assay for muscle-specific tyrosine kinase (MuSK) autoantibodies in myasthenia gravis. J. Neuroimmunol. 240-241, 79-86. 
Tsiamalos, P., Kordas, G., Kokla, A., Poulas, K., Tzartos, S.J., 2009. Epidemiological and immunological profile of muscle-specific kinase myasthenia gravis in Greece. Eur. J. Neurol. 16, 925-930.

Vincent, A., Drachman, D.B., 2002. Myasthenia gravis. Adv. Neurol. 88, 159-188.

Vincent, A., Bowen, J., Newsom-Davis, J., McConville, J., 2003. Seronegative generalised myasthenia gravis: clinical features, antibodies, and their targets. Lancet Neurol. 2, 99-106.

Vincent, A., Leite, M.I., Farrugia, M.E., Jacob, S., Viegas, S., Shiraishi, H., et al., 2008 Myasthenia gravis seronegative for acetylcholine receptor antibodies. Ann. N. Y. Acad. Sci. 1132, 84-92.

Vincent, A., Waters, P., Leite, M.I., Jacobson, L., Koneczny, I., Cossins, J., et al., 2012. Antibodies identified by cell-based assays in myasthenia gravis and associated diseases. Ann. N. Y. Acad. Sci. 1274, 92-98.

Yamamoto, T., Vincent, A., Ciulla, T.A., Lang, B., Johnston, I., Newsom-Davis, J., 1991. Seronegative myasthenia gravis: a plasma factor inhibiting agonist-induced acetylcholine receptor function copurifies with IgM. Ann. Neurol. 30, 550-557.
Yang, L., Maxwell, S., Leite, M.I., Waters, P., Clover, L., Fan, X., et al., 2011. Non-radioactive serological diagnosis of myasthenia gravis and clinical features of patients from Tianjin, China. J. Neurol. Sci. 301, 71-76.

Zhang, B., Tzartos, J.S., Belimezi, M., Ragheb, S., Bealmear, B., Lewis, R.A., et al., 2012. Autoantibodies to lipoprotein-related protein 4 in patients with double-seronegative myasthenia gravis. Arch. Neurol. 69, 445-451.

Zisimopoulou, P., Evangelakou, P., Tzartos, J., Lazaridis, K., Zouvelou, V., Mantegazza, R., et al., 2013. A comprehensive analysis of the epidemiology and clinical characteristics of anti-LRP4 in myasthenia gravis. J. Autoimmun. 52, 139-145.

Zouvelou, V., Kyriazi, S., Rentzos, M., Belimezi, M., Micheli, M.A., Tzartos, S.J., et al., 2013. Double-seropositive myasthenia gravis. Muscle Nerve 47, 465-466. 